\title{
Mitteilungen der DGMKG
}

MKG-Chirurg 2020 - 13:303-305

https://doi.org/10.1007/s12285-020-00273-3

(c) Springer Medizin Verlag GmbH, ein Teil von Springer Nature 2020

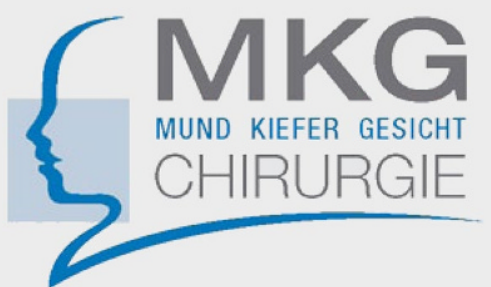

\author{
Redaktion \\ Dr. Jörg-Ulf Wiegner, Saalfeld \\ (v.i.S.d.P.)
}

Korrespondenzadresse

Deutsche Gesellschaft für Mund-, Kiefer- und Gesichtschirurgie e.V. Geschäftsstelle

Geschäftsführerin Kerstin Kothe

Schoppastraße 4, 65719 Hofheim

Tel. 06192-20 63 03, Fax 06192-20 6304

E-Mail: info@dgmkg.de

Internet: www.dgmkg.de

\section{Inhalt}

303 Ein Rückblick auf 70 Jahre DGMKG in Bild und Ton

304 Zur rechtlichen Einordnung von Leitlinien

305 Veranstaltungen

\section{Ein Rückblick auf 70 Jahre DGMKG in Bild und Ton}

Sehr geehrte Kolleginnen und Kollegen,

unsere Deutsche Gesellschaft für Mund-, Kiefer- und Gesichtschirurgie (DGMKG) hätte im Juni dieses Jahres ihre 70. Jahrestagung in Hamburg durchgeführt. Wegen der Corona-Krise hat sich der Vorstand der DGMKG entschieden, den Kongress 2020 ausfallen zu lassen. Um das Jubiläum trotz ausgefallener Jahrestagung zu begehen, haben wir Fachvertreter unserer Gesellschaft gebeten, die vergangenen drei Epochen Revue passieren zu lassen und uns einen Ausblick auf die Zukunft zu geben. Das Besondere: Die Vorträge von Dr. Lutz Tischendorf, Prof. Karsten Gundlach, Dr. Hans-Peter Ulrich und Prof. Jürgen Hoffmann wurden in Bild und Ton festgehalten (s. Kasten).

Einen ersten kurzen Einblick in die lebhaften vergangenen 70 Jahre der MKG-Chirurgie in Deutschland möchte ich Ihnen im Folgenden geben.

In der ehemaligen DDR gab es eine hoch entwickelte Mund, Kiefer- und Gesichtschirurgie.
So fand z.B. die erste Großzehentransplantation als Daumenersatz in Jena, durchgeführt von Prof. Dieter Schumann, statt. Das Generationen von Studierenden wie auch Kolleg(inn)en bekannte Lehrbuch Zahn-Mund-Kiefer-Heilkunde. Lehrbuchreihe zur Aus- und Weiterbildung wurde von Prof. Norbert Schwenzer (Tübingen) und Prof. Gerhard Grimm (Halle/Saale) verfasst und zeigt, dass es trotz der Teilung Deutschlands eine erfolgreiche Zusammenarbeit gab. Nicht unerwähnt bleiben soll auch das international bekannte Spaltzentrum der DDR in Thallwitz bei Leipzig. Kollege Dr. Lutz Tischendorf aus Halle hat diese Epoche der MKG-Chirurgie nicht nur erlebt, sondern auch aktiv gestaltet und von seiner Warte aus einen Vortrag über diese Zeit gehalten. Seien Sie gespannt!

Prof. Karsten Gundlach hat diese Zeit im westlichen Teil Deutschlands erlebt. Er war lange Zeit in Hamburg tätig, zunächst als Assistent und später als Oberarzt. Prof. Karsten Gundlach war u.a. Schüler von Prof. Karl Schuchardt, bevor er nach der Wiedervereinigung Deutschlands Ordinarius in Rostock wurde. Freuen Sie sich auf seine Erfahrungen mit der westdeutschen MKG-Chirurgie zu dieser Zeit.

Nach der Wiedervereinigung Deutschlands 1989 wurden dann die DGMKG und der Berufsverband der Mund-, Kiefer- und Gesichtschirurgen zusammengeführt. Diesen Zusammenschluss sahen beide Seiten anfangs z.T. nicht unkritisch. Wer kann diese Epoche besser beschreiben als Dr. Hans-Peter Ulrich. Er war in diesen unruhigen Zeiten 2-mal Präsident der DGMKG und hat maßgeblich dazu beigetragen, die Gesellschaft in ein ruhiges Fahrwasser zu bringen. Herr Kollege Ulrich wird seine Eindrücke sicherlich kurzweilig und pointiert darstellen.

Letztlich hat sich dieser $\mathrm{Zu}$ sammenschluss als Erfolgsmodell für die Zukunft entwickelt. Es sind vielfältige Einflussfaktoren, die jetzt und sicher auch in Zukunft von nationaler, europäischer und internationaler Seite auf die deutsche Mund-, Kiefer- und Gesichtschirurgie Einfluss nehmen. Prof. Jürgen Hoffmann aus Heidelberg hat es sich zur Aufgabe gemacht, die Chancen, Perspektiven und Risiken für unser Fach in seinem Vortrag darzustellen.

Lieber Herr Kollege Tischendorf, lieber Karsten, lieber Herr Kollege Ulrich, lieber Jürgen,

ganz herzlichen Dank für Eure Bereitschaft, Euch für die Deutsche Mund-, Kiefer- und Gesichtschirurgie mit Euren Vorträgen zu engagieren. Wir dürfen ein facettenreiches, nie langweiliges und auch herausforderndes Fach vertreten, das auch für die Zukunft dank unserer Vorväter und jetzigen Protagonist(inn)en zukunftssicher aufgestellt ist. Ich freue mich, Teil dieser Gemeinschaft sein zu dürfen.

Mit kollegialen Grüßen

\section{Prof. Dr. Dr. Jörg Wiltfang} Klinik für Mund-, Kiefer- und Gesichtschirurgie Universitätsklinikum SchleswigHolstein, Kiel

\section{Ein Rückblick auf 70 Jahre DGMKG in Bild und Ton}

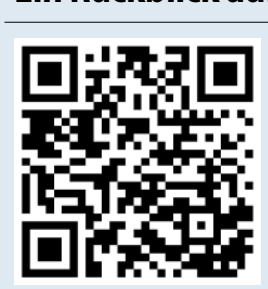

Die Videoaufnahmen der Vorträge zum Jubiläum inklusive einführender Worte durch den DGMKG-Präsidenten finden Sie ab November im geschützten Mitgliederbereich auf der Homepage der DGMKG (https://www. dgmkg.com/dgmkg-intern). 


\section{Zur rechtlichen Einordnung von Leitlinien}

Anlässlich der Corona-Pandemie hatte die Arbeitsgemeinschaft der Wissenschaftlichen Medizinischen Fachgesellschaften (AWMF) jüngst „Interdisziplinär abgestimmte Empfehlungen zum Personal- und Patientenschutz bei Durchführung planbarer Eingriffe zur Zeit der SARSCoV-2-Pandemie" im Status einer S1-Leitlinie veröffentlicht, die sich (auch) mit der präoperativen COVID-19-Testung der Patienten vor ambulanten Eingriffen beschäftigt. Diese Leitlinie hat mancherorts die Frage aufgeworfen, welche rechtliche Bedeutung Leitlinien eigentlich haben.

Hierzu wie folgt: Leitlinien werden typischerweise von ärztlichen und/oder zahnärztlichen Fachgesellschaften unter dem Dach der AWMF entwickelt und publiziert. Da die AWMF keine staatliche Organisation, sondern ein privatrechtlicher Verein ist, handelt es sich bei den Leitlinien aber nicht um Instrumente der Rechtsetzung, wie dies etwa bei den vom Gemeinsamen Bundesausschuss (G-BA) veröffentlichten Richtlinien der Fall ist. Daher haben sie - anders als die Richtlinien des G-BA - keine unmittelbare rechtliche Verbindlichkeit. In der medizinrechtlichen Praxis haben sie insoweit Relevanz, als sie bei Streitigkeiten über Behandlungsfehler häufig mit herangezogen werden, um den ärztlichen Standard zum Zeitpunkt der Behandlung zu definieren. Zur Erinnerung: Ein Behandlungsfehler liegt vor, wenn der Behandler von den zur Zeit der Behandlung anerkannten fachlichen Standards abweicht, ohne dass eine Abweichung entsprechend mit dem Patienten vereinbart worden war ( $\$ 630$ a Abs. 2 Bürgerliches Gesetzbuch). Mithin ist für die Frage nach dem Behandlungsfehler die Bestimmung des ärztlichen Standards unerlässlich. Nach der Rechtsprechung des Bundesgerichtshofs
(BGH; Urteil vom 15. April 2014 - VIZR 382/12) dürfen aber Leitlinien nicht unbesehen mit dem medizinischen Standard gleichgesetzt werden. Sie ersetzen insbesondere kein Sachverständigengutachten im Prozess. Zwar - so der BGH - können Leitlinien im Einzelfall den medizinischen Standard für den Zeitpunkt ihres Erlasses zutreffend beschreiben; sie können aber auch aufgrund der stetigen Fortentwicklung der Medizin veralten. Mithin sind sie Momentaufnahmen. Aus diesem Grunde kann sich der Tatrichter, dem die Ermittlung des ärztlichen Standards im Einzelfall obliegt, nicht allein auf Leitlinien stützen. Vielmehr ist er gehalten, eine Bewertung des Behandlungsgeschehens durch einen Sachverständigen aus dem betroffenen medizinischen Fachgebiet vornehmen zu lassen. Üblicherweise wird dieser Sachverständige sodann aber nicht umhinkommen, bei der Bestimmung des (zahn-)ärztlichen Standards zum Zeitpunkt der Behandlung auch die hierzu publizierten Leitlinien zu würdigen.

Wenngleich Leitlinien also rechtlich unverbindlich sind und nicht ohne Weiteres mit dem medizinischen Standard gleichgesetzt werden können, wird jeder Behandler dennoch gut daran tun, sie im Blick zu behalten und im Einzelfall zu überlegen, inwieweit eine Abweichung von den in den Leitlinien vorgegebenen Handlungsempfehlungen medizinisch zu vertreten ist.

Detaillierte Ausführungen zur rechtlichen Bedeutung von Leitlinien finden Sie im Übrigen im CME-Beitrag „Rechtliche Bedeutung medizinischer Leitlinien" in Ausgabe 2/2016 von Der MKG-Chirurg (s. weiterführende Literatur).

\section{Weiterführende Literatur}

- Müller M, Ulrich H-P (2016) Rechtliche Bedeutung medizinischer Leitlinien. MKGChirurg; 9(2):131-141; https://doi.org/10.1007/ s12285-016-0050-9

\section{Prof. Dr. Matthias Müller} Schick und Schaudt Rechtsanwälte PartG mbB, Stuttgart

Sehr geehrte Kolleginnen und Kollegen,

zuerst gilt unser Dank dem Justiziar der DGMKG, Herrn Prof. Matthias Müller, für die schnelle Bearbeitung des Themas.

Wie ausgeführt, hat dieses bereits 2016 seinen Niederschlag in Der MKG-Chirurg gefunden. Wegen der Aktualität wird das Thema jetzt in Kurzversion erneut aufgegriffen und die rechtliche Lage von Leitlinien dargestellt.

Bedauerlicherweise ist es rechtlich ohnehin so, dass die Entscheidungen dem Behandler/Operateur nicht abgenommen werden können und damit natürlich auch nicht die Haftung. Das bedeutet im Umkehrschluss, wenn eine Leitlinie einmal in der Welt ist, haben wir damit zu leben. Klug ist es, sich im Vorfeld so einzubringen, dass keine für uns negativen Entwicklungen auftreten, selbstverständlich un- ter gleichzeitiger Beachtung des Schutzes der uns anvertrauten Patienten und unter Einbeziehung des medizinischen Fortschritts.

Deshalb sollten wir sowohl den Text von Herrn Prof. Müller als auch die hier angesprochene S1-Leitlinie der AWMF zum Anlass nehmen, für die Mitarbeit bei der Erstellung von Leitlinien zu werben - eine hoch verantwortungsvolle Tätigkeit für uns alle. Gleichzeitig gilt unser Dank denjenigen, die sich über Jahre intensiv an der Leitlinienarbeit beteiligt haben. Die DGMKG bringt sich in den Novellierungsprozess der genannten S1-Leitlinie der AWMF mit Herrn Prof. Bilal AlNawas aktiv ein, um den facettenreichen Aspekten der MKG-chirurgischen Patientenversorgung gerecht zu werden

Es wird, dafür bitten wir um Verständnis, natürlich nicht immer gelingen, alle möglichen Eventualitäten einer Leitlinie so zu bedenken, dass jeder damit zufrieden ist. Es bleibt „work in progress".

Ich hoffe, dass es Ihnen allen gut geht und dass Sie gesund bleiben. Bleiben Sie uns gewogen! Viele Grüße

\section{Dr. Dr. Hans-Peter Ulrich} MKG-Chirurgie LindenArcaden Lübeck

\section{Die DGMKG ist wie folgt für Sie erreichbar}

Geschäftsführerin Frau Kerstin Kothe

Schoppastraße 4, 65719 Hofheim

Tel. 06192-206303, Fax 06192-206304

E-Mail: info@dgmkg.de

Internet: www.dgmkg.de 


\section{Veranstaltungen}

Wiesbaden, 05.-06.02.2021
MKG-Update 2021
12. MKG-Update-Seminar
Unter der Schirmherrschaft
der DGMKG
Wiss. Leitung:
Prof. Dr. Dr. Knut A. Grötz
Prof. Dr. Andrea M. Schmidt-
Westhausen
Prof. Dr. Dr. Stefan Haßfeld
www.mkg-update.com
Infos zu allen Update-Seminaren:
med update GmbH
Tel.: 0611 - 73 65 80
Fax: 0611 - 73 65 810
info@med-update.com
Partner in der Fortbildung
$\quad$ med Springer Medizin
$\quad$ mpdate
med

Sehr geehrte Mitglieder, hiermit möchten wir Sie über kommende Veranstaltungen informieren.

\section{Veranstaltungen der DGMKG}

München, 11.-12.12.2020

Implantologie für den Praktiker/Gutachtertagung

25.02.2021*

Fachkunde Strahlenschutz im Rahmen des Praxisführungsseminars

26.-28.02.2021*

Praxisführungsseminar DGMKG

Düsseldorf, 19.-21.03.2021

Curriculum Implantologie DGMKG/BDO

Düsseldorf, 19.-21.03.2021 Curriculum Parodontologie DGMKG/BDO

*Veranstaltungsort wird noch bekannt gegeben (s. Veranstaltungskalender auf der DGMKG-Homepage).
München, 14.-17.04.2021

71. Kongress der DGMKG im Rahmen des DGCh-

Kongresses

Hamburg, 12.-14.11.2021

Curriculum Implantologie DGMKG/BDO

Hamburg, 12.-14.11.2021

Curriculum Parodontologie DGMKG/BDO

München, 10.-11.12.2021

Implantologie für den

Praktiker

\section{Veranstaltungen von Mitgliedern}

Berlin, 02.12.2020

Fachkunde Dentale Volumentomographie 2. Teil

München, 03.-05.12.2020

67th International Course for Stepwise Flap Raising

Aachen, 18.-20.02.2021 68th International Course for Stepwise Flap Raising

Aachen, 21.-23.02.2021

19. EUREGIO Implantationskurs am Humanpräparat

Bochum, 10.-19.03.2021

69th International Course for Flap Raising \& Microsurgery

\section{Weitere Veranstaltungen}

Aachen, 14.-17.11.2020

51. DÖSAK-Tagung

Maastricht, 31.05.-04.06.2021

TERMIS World Congress

Weitere Informationen und Anmeldeformulare finden Sie auf der DGMKG-Homepage im Veranstaltungskalender unter: https://www.dgmkg.de/ veranstaltungen/ 